\title{
Qualitative analysis of two-fluid FRW cosmological models
}

\author{
A.K. Verma
}

Received: 9 November 2008 / Accepted: 12 February 2009 / Published online: 26 February 2009

(c) The Author(s) 2009. This article is published with open access at Springerlink.com

\begin{abstract}
This paper focuses attention on a qualitative analysis of the evolution of two-fluid flat FRW cosmological models.In the first model one of the fluid represents matter content of the universe comoving with respect to the another fluid that is the cosmic microwave background radiation (CMBR), these two fluids are interacting. The first model is most relevant to describe the scenario before the recombination epoch when matter and radiation were in an interactive phase and the photons was bound to electron through Thomson scattering. The second model describe two noninteracting fluids where the matter is comoving to the space-time coordinates and the CMBR is moving axially, relative to the matter thus modeling the relative velocity between galaxies and the CMBR (Phys. Rev. Lett. 39:898901, 1977). This model portray the cosmic evolution in the postrecombination epoch when the two-fluid are noninteracting.In this epoch the photons got themselves free to form the CMBR being observed presently.
\end{abstract}

Keywords Cosmic microwave background radiation . Hubble parameter - Equilibrium point - Bendixson criteria . Limit cycles $\cdot$ Attractor

PACS 04.20.Jb $\cdot$ 98.80.Dr $\cdot 98.80 . \mathrm{Hw} \cdot 98.80 . \mathrm{K}$

\section{Introduction}

In the existing epoch the perceptible universe is consist of extremely isotropic background radiation in the mi-

A.K. Verma (凶)

Department of Mathematical Sciences and Computer

Applications, Bundelkhand University, Jhansi 284128,

Uttar Pradesh, India

e-mail: alokverma_bu@yahoo.com crowave region (CMBR) and the incoherent matter in the form of galactic conglomeration following the Hubble flow. Since the universe at large is spatially homogeneous and isotropic, the background geometry is close to that of Robertson-Walker (R-W) space-time with zero or constant (positive or negative) spatial curvature. Therefore Friedmann-Robertson-Walker (FRW) spatially homogeneous and isotropic models are widely regarded as acceptable approximation of the existing and primeval phases of the universe.

The cosmic evolution based on two-fluid big-bang model appears better than two sequence of a single fluid model. Our object is to present two-fluid model with background geometry that of Robertson-Walker space-time. In these models we assume that both the fluids are present throughout the cosmic evolution and one fluid dominating the other. This type of model can be used to describe, the transition between a radiation dominated phase to a matter dominated phase as the universe evolves.

In the two-fluid model if the fluids are interacting then they satisfy unified conservation law. The law of conservation of energy and momentum is given by

$\left(T_{j ; i}^{i}\right)_{m}+\left(T_{j ; i}^{i}\right)_{r}=0$,

where

$T_{j ; i}^{i}=\frac{\partial}{\partial x^{i}} T_{j}^{i}+\Gamma_{i k}^{i} T_{j}^{k}-\Gamma_{i j}^{k} T_{k}^{i}$.

When fluids are noninteracting then corresponding energy and momentum are conserved separately.

$$
\begin{aligned}
& \left(T_{j ; i}^{i}\right)_{m}=0 \\
& \left(T_{j ; i}^{i}\right)_{r}=0,
\end{aligned}
$$


here the non tensor suffixes $m$ and $r$ denote fluid parameters of matter and radiation respectively.

Coley and Wainwright (1992) discussed the noninteracting case based on dynamical systems approach while we assume that both fluids are interacting. Coley and van den Hoogen (1995) has presented qualitative analysis of a single fluid FRW space-time with non vanishing bulk viscosity.

If a perfect fluid space-time is self similar (Coley and Tupper 1989) that is it admits a homothetic vector field $\xi^{i}$ (say) then the Lie derivative of the density, pressure and fluid velocity with respect to $\xi^{i}$ is not zero, in other words the quantities inherit space-time symmetry defined by the homothetic vector field $\xi^{i}$. It has been proved by Coley and Tupper in symmetry inheritance Theorems 1-6 (Coley and Tupper 1989) that a viscous fluid space-time can also inherit space-time symmetry associated with a homothetic vector field. Therefore a non maximally symmetric energymomentum tensor can give rise to a maximally symmetric space-time. In fact it has been further shown by Coley and Tupper in their paper (1989) that FRW cosmologies do not necessarily represent perfect fluid solutions but can also be exact solution of the field equation for a viscous fluid in a frame non comoving to the coordinate of $\mathrm{R}-\mathrm{W}$ metric.The various imperfect FRW cosmologies have been discussed in details by Coley and Tupper (1983, 1984, 1985, 1986). In the second model we shall discuss imperfect FRW cosmologies where galactic matter is represented by a comoving perfect fluid and CMBR is modeled as a tilted, imperfect fluid having finite shear viscosity and vanishing bulk viscosity.

\section{Model (i). Two perfect-fluid model}

In this model we assume that the two-fluid are comoving, interacting and obey a $\gamma$-law for the equation of state. The total density and pressure are $\rho=\rho_{r}+\rho_{m}, p=p_{r}+p_{m}$, respectively where

$p_{r}=\left(\gamma_{r}-1\right) \rho_{r}, \quad p_{m}=\left(\gamma_{m}-1\right) \rho_{m}$,

here suffixes $r$ and $m$ and denote fluid parameters of radiation and matter respectively, $\gamma_{r}, \gamma_{m}$ are constants with

$\gamma_{r} \neq \gamma_{m}$

The flat Robertson-Walker line element in the axial coordinates is given by

$d s^{2}=d t^{2}-R^{2}(t)\left[d x^{2}+d y^{2}+d z^{2}\right]$,

the four-velocity of the two-fluid that is matter and radiation are given by

$u_{i}=(1,0,0,0), \quad v_{i}=(1,0,0,0)$.
Equations of general relativity are

$R_{i j}-\frac{1}{2} R g_{i j}=-T_{i j}$

where

$$
\begin{aligned}
T_{i j}= & \gamma_{r} \rho_{r} u_{i} u_{j}-\left(\gamma_{r}-1\right) \rho_{r} g_{i j}+\gamma_{m} \rho_{m} v_{i} v_{j} \\
& -\left(\gamma_{m}-1\right) \rho_{m} g_{i j} .
\end{aligned}
$$

The Einstein field equations are

$\frac{\dot{R}^{2}}{R^{2}}+\frac{2 \ddot{R}}{R}=-\left(\left(\gamma_{r}-1\right) \rho_{r}+\left(\gamma_{m}-1\right) \rho_{m}\right)$,

$\frac{3 \dot{R}^{2}}{R^{2}}=\rho_{r}+\rho_{m}$,

and the energy conservation equation is given by

$\dot{\rho}_{r}+\dot{\rho}_{m}+3 \frac{\dot{R}}{R}\left(\gamma_{r} \rho_{r}+\gamma_{m} \rho_{m}\right)=0$.

The Hubble parameter is given by

$H=\frac{\dot{R}}{R}$.

In order to write field equations in dimensionless form we introduce the density parameter

$\Omega=\frac{\rho}{3 H^{2}}$,

$\Omega=\Omega_{m}+\Omega_{r}$,

and the deceleration parameter

$q=-\frac{\ddot{R}}{\dot{R}^{2}} R$.

Let a dimensionless time variable $\tau$ (Wainwright 1997) related to the scale factor $R$ is given by

$R=R_{0} e^{\tau}$

where $R_{0}$ is the value of the scale factor at some arbitrary reference time. It follows from (10) that

$\frac{d t}{d \tau}=\frac{1}{H}$

from (10), (13) and (14) (Wainwright 1997)

$H^{\prime}=-(1+q) H$,

where prime denotes differentiation with respect to $\tau$, from (7), (10), (11) and (13)

$2 q=3\left[\gamma_{r} \Omega_{r}+\gamma_{m} \Omega_{m}-\Omega\right]+1$. 
In view of (10), (11), (15)-(17) the conservation equation (9) reduces to

$\Omega^{\prime}-3[\Omega-1][\mu-\Omega]=0$,

where $\mu=\gamma_{r} \Omega_{r}+\gamma_{m} \Omega_{m}$, hyperbolic equilibrium points are given by

$\Omega=1, \quad \Omega=\mu$.

These equilibrium points lie on the curve

$\Omega^{2}-\Omega(1+\mu)+\mu=0$,

we rewrite (18) as

$f(\Omega, \mu) \equiv \Omega^{\prime}=3[\Omega-1][\mu-\Omega]$,

$\left(\frac{\partial f}{\partial \Omega}\right)_{\Omega=1}=3[\mu-1]$,

$\left(\frac{\partial f}{\partial \Omega}\right)_{\Omega=\mu}=3[1-\mu]$.

The equilibrium points $\Omega=1$ is stable(unstable) and $\Omega=\mu$ is unstable (stable) provided $\mu<1(\mu>1)$. Integrating (18)

$\Omega=\frac{\mu-a e^{3(\mu-1) \tau}}{1-a e^{3(\mu-1) \tau}}$,

were $a$ is the constant of integration.

Case (i) $\mu<1$

When $\tau \rightarrow-\infty \Rightarrow \Omega \rightarrow \mu$ and whereas $\tau \rightarrow \infty \Rightarrow \Omega \rightarrow 1$.

Here $\Omega=\mu$ and $\Omega=1$ are the $\alpha$ and $\omega$ limit points of the trajectories of (18).

Case (ii) $\mu>1$

When $\tau \rightarrow-\infty \Rightarrow \Omega \rightarrow 1$ and whereas $\tau \rightarrow \infty \Rightarrow \Omega \rightarrow \mu$. In this case $\Omega=1$ and $\Omega=\mu$ are the $\alpha$ and $\omega$ limit points of the trajectories of (18). These are also asymptotically stable equilibrium points and the paths around them do not formed closed curves, $\Omega=1$ corresponds to flat FRW universe.

Attractors: Attracters are defined as a closed invariant set of a differential equation which contains a dense orbit (Perko 1991; Wainwright 1997). An invariant set $\Phi \subset R^{n}$ for (18) is defined by

$\Phi=\{\Omega \mid \mu \leq \Omega \leq 1\}$,

is an attractor for (18).

\section{Model (ii). Perfect matter field and viscous CMBR}

In this section we shall discuss noninteracting two-fluid FRW model in which the matter distribution is assumed to be a perfect fluid and CMBR is modeled as a viscous fluid having finite shear viscosity. Here coordinates are comoving with the matter, that is the four-velocity of the matter is given by

$v_{i}=(1,0,0,0)$,

and

$$
\begin{aligned}
T_{i j}= & \left(\rho_{m}+p_{m}\right) v_{i} v_{j}-p_{m} g_{i j}+\frac{\rho_{r}}{3}\left(4 u_{i} u_{j}-g_{i j}\right) \\
& -2 \eta \sigma_{i j}+q_{i} u_{j}+q_{j} u_{i} .
\end{aligned}
$$

Here $u_{i}$ is the time-like unit four-velocity of tilted CMBR and has a non zero spatial component.

$u_{i}=(\alpha, 0,0,-\beta R), \quad \alpha^{2}-\beta^{2}=1$,

$\beta \mathrm{R}$ accounts for the deviation of CMBR from the Hubble flow. The physical and kinematical quantities associated with the model are functions of $t$ alone. $\eta$ is the shear viscosity coefficient of CMBR and $\sigma_{i j}$ the shear tensor

$\sigma_{i j}=\frac{1}{2}\left[u_{i ; \gamma} H_{j}^{\gamma}+u_{j ; \gamma} H_{i}^{\gamma}\right]-\frac{1}{3} u_{; \gamma}^{\gamma} H_{i j}$,

where

$H_{i j}=u_{i} u_{j}-g_{i j}$

$q_{i}$, the heat conduction vector, satisfies the condition

$q_{i} u^{i}=0$.

In view of (27) a solution of (30) is given by

$q_{i}=Q(-\beta, 0,0, \alpha R), \quad Q^{2}=q_{i} q^{i}$.

The independent field equations are

$\frac{\dot{R}^{2}}{R^{2}}+\frac{2 \ddot{R}}{R}=-\left(\frac{\rho_{r}}{3}+p_{m}+\frac{2 \eta \beta \dot{\beta}}{3 \sqrt{1+\beta^{2}}}\right)$,

$\frac{-3 \dot{R}^{2}}{R^{2}}=\left(\frac{\rho_{r}}{3}\left(3+4 \beta^{2}\right)+\rho_{m}+\frac{4 \eta \beta \dot{\beta}\left(3+2 \beta^{2}\right)}{3 \sqrt{1+\beta^{2}}}\right)$,

$\rho_{r}=-\frac{\eta \dot{\beta}\left(3+4 \beta^{2}\right)}{2 \beta \sqrt{1+\beta^{2}}}$.

For the equation of state of matter we shall assume the $\gamma$-law

$p_{m}=(\gamma-1) \rho_{m}$.

From (32)-(35) we obtain a second order nonlinear differential equation in $R$ alone (Jacob 1967)

$\ddot{R} R+\dot{R}^{2}-\frac{(4-3 \gamma)}{6} \rho_{m 0} R_{0}^{3 \gamma} R^{2-3 \gamma}=0$. 
The first integral is

$\dot{R}^{2}=\frac{\rho_{m 0} R_{0}^{3 \gamma} R^{2-3 \gamma}}{3}+\frac{b}{R^{2}}$,

$b$ being constant of integration. From (33) and (34) we obtain

$\Omega_{m}+\frac{\Omega_{r}}{1+\frac{4 \beta^{2}}{3}}=1$,

where

$\Omega_{m} \equiv \frac{\rho_{m}}{3 H^{2}}, \quad \Omega_{r} \equiv \frac{\rho_{r}}{3 H^{2}}$,

denote respectively the matter density parameter and the radiation density parameter and

$H=\frac{\dot{R}}{R}$

is the Hubble parameter. The two-fluid components of the distribution are noninteracting so that we have separate conservation equations

$\dot{\rho}_{m}+3 \gamma \rho_{m} \frac{\dot{R}}{R}=0$,

$\dot{\rho}_{r}+\rho_{r}\left[\frac{4 \dot{R}}{R}-\frac{8 \beta \dot{\beta}}{3+4 \beta^{2}}\right]=0$,

out of which one equation is independent. From (33), (34), (37) and (41) we obtain

$\frac{\eta \dot{\beta}}{\beta \sqrt{1+\beta^{2}}}+\frac{3 b}{R^{4}}=0$.

Eliminating $t$ between (37) and (43) one can derive

$\frac{\eta d \beta}{\beta \sqrt{1+\beta^{2}}}+\frac{a_{1} d R}{R^{3} \sqrt{a_{2}^{2}+R^{4-3 \gamma}}}=0$,

with

$a_{1} \equiv \frac{3 b^{3 / 2}}{a_{2}}, \quad a_{2} \equiv\left(\frac{3 b}{\rho_{m 0} R_{0}^{3 \gamma}}\right)^{1 / 2}$.

Thus we have five independent equations (32)-(35) and (41) in six variables $\rho_{m}, p_{m}, \rho_{r}, \beta, \eta$ and $R$. For a deterministic system of equations we require one more equation for which we assume that

$R=R_{0} e^{-\beta}$,

where $R_{0}$ is the value of the scale factor at some arbitrarily chosen reference time. We obtain
Our aim is to write the equations governing the dynamics of the system with respect to the dimensionless independent variable $\beta$. The deceleration parameter can be expressed in the form

$q=\frac{H^{\prime}}{H}-1$

where prime denotes differentiation with respect to $\beta$. From (32)-(35) we obtain

$q=1+\left(\frac{3 \gamma}{2}-2\right) \Omega_{m}=\frac{3 \gamma}{2}-1+\frac{3(4-3 \gamma)}{2\left(3+4 \beta^{2}\right)} \Omega_{r}$.

We put (41) and (42) in the form

$\dot{\Omega}_{m}=\frac{a_{1}(4-3 \gamma)\left(1-\Omega_{m}\right) \Omega_{m}}{2 \overline{\Omega_{r}} R_{0}^{2} e^{-2 \beta} \sqrt{a_{2}^{2}+R_{0}^{4-3 \gamma} e^{-(4-3 \gamma) \beta}}}$,

$\dot{\overline{\Omega_{r}}}=\frac{a_{1}(3 \gamma-4)\left(1-\overline{\Omega_{r}}\right)}{2 R_{0}^{2} e^{-2 \beta} \sqrt{a_{2}^{2}+R_{0}^{4-3 \gamma} e^{-(4-3 \gamma) \beta}}}$,

where

$\overline{\Omega_{r}} \equiv \frac{\Omega_{r}}{1+\frac{4}{3} \beta^{2}}$.

From (37), (43) and (46) we get

$\dot{\beta}=-\frac{a_{1}}{2 \overline{\Omega_{r}} R_{0}^{2} e^{-2 \beta} \sqrt{a_{2}^{2}+R_{0}^{4-3 \gamma} e^{-(4-3 \gamma) \beta}}}$,

from (44) and (46)

$\eta=\frac{a_{1} \beta \sqrt{1+\beta^{2}}}{R_{0}^{2} e^{-2 \beta} \sqrt{a_{2}^{2}+R_{0}^{4-3 \gamma} e^{-(4-3 \gamma) \beta}}}$,

we rewrite (50) and (51) as

$g\left(\Omega_{m}, \overline{\Omega_{r}}\right) \equiv \dot{\Omega}_{m}$

$$
\begin{aligned}
& =\frac{a_{1}(4-3 \gamma)\left(1-\Omega_{m}\right) \Omega_{m}}{2 \overline{\Omega_{r}} R_{0}^{2} e^{-2 \beta} \sqrt{a_{2}^{2}+R_{0}^{4-3 \gamma} e^{-(4-3 \gamma) \beta}}} \\
h\left(\Omega_{m}, \overline{\Omega_{r}}\right) & \equiv \dot{\Omega_{r}}=\frac{a_{1}(3 \gamma-4)\left(1-\overline{\Omega_{r}}\right)}{2 R_{0}^{2} e^{-2 \beta} \sqrt{a_{2}^{2}+R_{0}^{4-3 \gamma} e^{-(4-3 \gamma) \beta}}} \\
\operatorname{div}[g, h] & =\frac{\partial g}{\partial \Omega_{m}}+\frac{\partial h}{\partial \bar{\Omega}_{r}}, \\
\operatorname{div}[g, h] & =\left[\frac{1-2 \Omega_{m}+\overline{\Omega_{r}}}{\overline{\Omega_{r}}}\right] .
\end{aligned}
$$

In view of (38) and (52)

$\operatorname{div}[g, h]=\left[2-3 \Omega_{m}\right]$ 
$\operatorname{div}[g, h]>0$ or $\operatorname{div}[g, h]<0$ provided $\Omega_{m}<2 / 3$ or $\Omega_{m}>$ $2 / 3$. $\operatorname{div}[g, h]$ is always positive or negative if $\Omega_{m}$ is less or greater than $2 / 3$, therefore from Bendixson's criteria (Perko 1991) for planar dynamical systems we conclude that the system of equations (50) and (51) has no closed orbits. From (50) and (51) one can obtain an ordinary differential equation

$\frac{d \Omega_{m}}{d \overline{\Omega_{r}}}=-\frac{\left(1-\Omega_{m}\right) \Omega_{m}}{\left(1-\overline{\Omega_{r}}\right) \overline{\Omega_{r}}}$.

In the phase plane $\left(\Omega_{m}, \overline{\Omega_{r}}\right)$ the curve given by (60) is not symmetrical. The path is vertical when they cross the curve $\left(1-\overline{\Omega_{r}}\right) \overline{\Omega_{r}}=0$ and it becomes horizontal as they cross the curve $\left(1-\Omega_{m}\right) \Omega_{m}=0$, by inspection of the sign of the right side of (50) shows that paths are directed to the right or left accordingly as $\Omega_{m}<1\left(\Omega_{m}>1\right)$ or $\Omega_{m}>1\left(\Omega_{m}<1\right)$ provided $\gamma>4 / 3(\gamma<4 / 3)$. From sign of the right side of (51) shows that paths move downward or upward accordingly as $\overline{\Omega_{r}}>1\left(\overline{\Omega_{r}}<1\right)$ or $\overline{\Omega_{r}}<1\left(\overline{\Omega_{r}}>1\right)$ provided $\gamma>4 / 3$ $(\gamma<4 / 3)$.

In view of (53), equations (50) and (51) can be expressed as uncoupled differential equations in $\Omega_{m}$ and $\overline{\Omega_{r}}$ respectively with the dimensionless quantity $\beta$ representing the independent variable

$\Omega_{m}^{\prime}+(4-3 \gamma)\left(1-\Omega_{m}\right) \Omega_{m}=0$,

${\overline{\Omega_{r}}}^{\prime}-(4-3 \gamma)\left(1-\overline{\Omega_{r}}\right) \overline{\Omega_{r}}=0$,

with the help of (38) and (52) the solution curves of (61) and (62) are respectively given by

$\Omega_{m}=\frac{1}{1+\frac{e^{(4-3 \gamma) \beta}}{c_{1}}}$,

$\overline{\Omega_{r}}=\frac{1}{1+c_{1} e^{-(4-3 \gamma) \beta}}$,

when $\beta \rightarrow-\infty \Rightarrow \Omega_{m} \rightarrow 1$ and $\overline{\Omega_{r}} \rightarrow 0$ whereas $\beta \rightarrow$ $\infty \Rightarrow \Omega_{m} \rightarrow 0$ and $\overline{\Omega_{r}} \rightarrow 1$. The equilibrium points $\Omega_{m}=$ $1, \Omega_{m}=0$ and $\overline{\Omega_{r}}=0, \overline{\Omega_{r}}=1$ respectively denote the $\alpha$, $\omega$ limit points of the trajectories of (61) and (62). Attractor for (61) and (62) are given by

$\Phi_{m}=\left\{\Omega_{m} \mid 0 \leq \Omega_{m} \leq 1\right\}$,

$\Phi_{r}=\left\{\overline{\Omega_{r}} \mid 0 \leq \overline{\Omega_{r}} \leq 1\right\}$,

where $\left|\Omega_{m}\right| \leq 1$ and $\left|\overline{\Omega_{r}}\right| \leq 1$ are the closed ball of radius unity with center at the origin. The hyperbolic equilibrium points of (61) and (62) in $\left(\Omega_{m}, \overline{\Omega_{r}}\right)$ plane are given by

$E_{1}=(0,0), \quad E_{2}=(0,1), \quad E_{3}=(1,1), \quad E_{4}=(1,0)$.
It can be shown that $E_{1}$ and $E_{3}$ are saddle points. The equilibrium points $E_{2}$ and $E_{4}$ respectively represent the source(sink) and sink(source) of the system for $\gamma>4 \backslash 3(<$ $4 \backslash 3)$. The bifurcation is brought about by the equation $\gamma=$ $4 \backslash 3$. We note that for $\gamma>4 \backslash 3, \beta \rightarrow-\infty \Rightarrow \Omega_{r} \rightarrow \infty$ and $\Omega_{m}=0$ whereas $\beta \rightarrow \infty \Rightarrow \Omega_{r}=0$ and $\Omega_{m}=1$. These results get swapped for $\gamma<4 \backslash 3$. $\dot{\beta}$ and $\eta$ are indeterminate as $\beta \rightarrow \pm \infty$.

\section{Conclusion}

We have presented two-fluid FRW cosmological models in which first one describe the two interacting perfect fluid model given by one dimensional autonomous nonlinear differential equation in $\Omega$, the overall density parameter. The second model describe noninteracting fluids with perfect fluid matter and imperfect CMBR. We have found that the dynamics of the distribution is described by two uncoupled differential equation in two dimensionless quantities using parameters of matter density $\Omega_{m}$ and a scalar multiple of radiation density $\overline{\Omega_{r}}$. In both the models their does not exist limit cycles that is path around the equilibrium points are not closed curves.

Open Access This article is distributed under the terms of the Creative Commons Attribution Noncommercial License which permits any noncommercial use, distribution, and reproduction in any medium, provided the original author(s) and source are credited.

\section{References}

Coley, A.A., Tupper, B.O.J.: Astrophys. J. 271, 1-8 (1983)

Coley, A.A., Tupper, B.O.J.: Astrophys. J. 280, 26-33 (1984)

Coley, A.A., Tupper, B.O.J.: Astrophys. J. 288, 418-421 (1985)

Coley, A.A., Tupper, B.O.J.: J. Math. Phys. 27, 406-416 (1986)

Coley, A.A., Tupper, B.O.J.: J. Math. Phys. 30, 2616-2625 (1989)

Coley, A.A., Wainwright, J.: Class. Quantum Gravit. 9, 651-665 (1992)

Coley, A.A., van den Hoogen, R.J.: Class. Quantum Gravit. 12, 19771994 (1995)

Jacob, K.C.: Nature 215, 1156-1157 (1967)

Perko, L.: Differential Equations and Dynamical Systems. Springer, New York (1991)

Smoot, G.F., Gorenstein, M.V., Muller, R.A.: Phys. Rev. Lett. 39, 898 901 (1977)

Wainwright, J.: In: Wainwright, J., Ellis, G.F.R. (eds.) Dynamical Systems in Cosmology. Cambridge University Press, Cambridge (1997) 